Real Analysis Exchange

Vol. 23(2), 1997-1998, pp. 421-430

S. J. Agronsky, Department of Mathematics, California Polytechnic State

University, San Luis Obispo, CA 93407, USA e-mail:

sagronsk@polymail . cpunix.calpoly.edu

J. G. Ceder, Department of Mathematics, University of California, Santa Barbara, CA 93106, USA

T. L. Pearson, Department of Mathematics and Statistics, Acadia University, Wolfville, Nova Scotia, B0P 1X0, Canada

\title{
SOME CHARACTERIZATIONS OF DARBOUX BAIRE 1 FUNCTIONS
}

\begin{abstract}
Two characterizations of Darboux Baire 1 functions are given: one in terms of pointwise limits of certain continuous functions, and the other in terms of intersections of sequences of open sets of a particular type.
\end{abstract}

\section{Introduction}

The main purpose of this article is to characterize Darboux Baire 1 functions from a closed interval into the reals as the pointwise limit of a sequence of polygonal functions, all having their vertices on the graph and such that the distances between consecutive vertices tend to zero (Theorem 3.1, below). In the process of doing this we obtain another characterization of a Darboux Baire 1 function in terms of its graph being the intersection of open strips (Theorem 2.4). These results answer questions posed in the survey paper [2]. Finally, if in Theorem 3.1 we drop the requirement that the distances between consecutive vertices tend to zero, we obtain a characterization of the Baire 1 functions (Theorem 4.1 below). In the originally submitted version of this paper, Theorem 4.1 was stated as a conjecture. We presented proofs that the condition was, indeed, necessary for several standard subclasses of the class of Baire 1 functions. The referee pointed out that both Theorem 4.1 and Theorem 3.1 can easily be obtained from a rather deep result in [3] concerning first return continuity. The referee's proofs are presented in Section 4. Our

Key Words: Darboux, Baire

Mathematical Reviews subject classification: 26A15

Received by the editors May 6, 1996 
technique for proving Theorem 3.1 is basically self-contained and, hopefully, will be found useful and insightful.

The procedure is to find a certain sequence of neighborhoods of $f$ (we do not distinguish $f$ from its graph) and in each such neighborhood we carefully construct a polygonal function whose vertices lie on $f$ and such that the distance between consecutive vertices is "small." Moreover, the sequence of neighborhoods has to be constructed so as to force the inserted polygonal functions to actually converge to $f$. By a polygonal function we mean a continuous function which is a union of finitely many line segments.

\section{Preliminaries}

Throughout this paper a fixed interval $I=[a, b]$ will be the domain of all the functions, unless otherwise noted. A set $G$ open relative to $I \times \mathbb{R}$ is called an open strip provided $\operatorname{dom} G=I$ and each vertical cross-section of $G$ is an open interval.

A Darboux function is one which maps intervals into intervals. A realvalued function $f$ on a metric space is Baire 1 iff $f^{-1}(G)$ is an $F_{\sigma}$-set whenever $G$ is open, iff $f$ is the pointwise limit of a sequence of continuous functions. A function $f$ is upper semicontinuous iff the associated sets $[f<\alpha]$ are open for every $\alpha \in \mathbb{R}$, iff $f$ is the pointwise limit of a monotonically decreasing sequence of continuous functions. (Lower semicontinuous (lsc) is defined as expected.) We denote by $\mathcal{D}, \mathcal{B}_{1}$, usc, and $\mathcal{C}$, respectively, the classes of Darboux, Baire 1 , upper semicontinuous, and continuous functions from $I$ into $\mathbb{R}$.

We say that $f$ is bilaterally dense in itself iff each open box having a point of $f$ in the interior of one of its vertical sides contains a point of $f$. Among Baire 1 functions, $f$ is Darboux iff $f$ is bilaterally dense in itself.

If $G$ is an open strip, we denote by $G(x)$ the intersection of $G$ with the vertical line through $x$. We say that a sequence $\left\{G_{n}\right\}_{n=1}^{\infty}$ of open strips is bilaterally dense in itself iff the set of vertical segments $\left\{G_{n}(x): x \in I, n=\right.$ $1,2, \ldots\}$ is bilaterally dense in itself, defined by replacing points by these segments in the definition of a bilaterally dense in itself function. The reader is referred to [2] for further facts about the class $\mathcal{D} \mathcal{B}_{1}$.

First we point out that an arbitrary open neighborhood of a given Darboux Baire 1 function $f$ need not contain a subneighborhood of $f$ which is an open strip. In fact if $f$ is not continuous, then $f$ has an open neighborhood not containing any open strips. To see this, suppose $\lambda$ is a cluster value of $f$ at $x_{0}$ different from $f\left(x_{0}\right)$. Supposing $f\left(x_{0}\right)<\lambda$ choose $2 \alpha=\lambda+f\left(x_{0}\right)$. Pick a sequence $\left\{x_{k}\right\}_{k=1}^{\infty}$ so that $\left(x_{k}, f\left(x_{k}\right)\right) \rightarrow\left(x_{0}, \lambda\right)$ with $f\left(x_{k}\right)>\alpha$ for each $k$. Then $(I \times \mathbb{R}) \backslash\left\{\left(x_{k}, \alpha\right): k=0,1,2, \ldots\right\}$ is an open neighborhood of $f$ which 
contains no open strip about $f$.

Although any open neighborhood of a Darboux Baire 1 function contains a continuous function (see [1]) and therefore by easy consequence a polygonal function, arbitrary open neighborhoods are too difficult to manage and we need to work with open strips instead.

To begin, the following result, although well-known and easy to prove, is presented here because our proof of Theorem 3.1 is a delicate elaboration of the basic theme of its proof, which we will only sketch.

Lemma 2.1. Each open strip is connected and contains a polygonal function with domain $I$.

Proof. Let $E$ consist of all $x \in I$ such that there exists a polygonal function $p$ from a fixed point $(a, c)$ in the open strip $G$ to some point $(x, y)$ such that $p \subseteq G$. It is easy to see that $E \neq \emptyset$. Let $\lambda=\sup E$. Next, show that $\lambda \in E$ using the openness of $G$ and the fact that vertical cross-sections of $G$ are intervals. Finally, show that $\lambda=b$ using the openness. (It is clear from the construction that one can construct a polygonal function $p$ in the strip to end at any prescribed point $(b, d)$.) Since $G$ is the union of $p$ with a set of vertical line segments, each intersecting $p, G$ is connected.

In the proof of Theorem 3.1 we will show how we can control the selection of the vertices in the above construction. We now proceed to construct an appropriate sequence of open strips which will squeeze down to a given Baire 1 function.

Although a Baire 1 function is a $G_{\delta^{-}}$set, not even a connected $G_{\delta}$-set need be Baire 1 (see [4]). However, Baire 1 functions can be characterized by $G_{\delta^{-}}$ sets of a certain type, as shown by the next result which is an extension of a result of Thomas [6] who proved it only for bounded functions. Our proof is more general and much simpler and is used specifically in Theorem 2.3.

Theorem 2.2. Let $f$ be a real-valued function on a metric space $X$. Then $f$ is Baire 1 if and only if $f$ is the intersection of a sequence of open strips.

Proof. $(\Rightarrow)$ Let $\left\{f_{n}\right\}_{n=1}^{\infty}$ be a sequence of continuous functions converging to $f$. Since $[h<g]$ is an $F_{\sigma}$-set whenever $h, g \in \mathcal{B}_{1}$, we can write

$$
\begin{aligned}
& {\left[f_{n}+\frac{1}{k}>f\right]=\bigcup_{i=1}^{\infty} A(n, k, i)} \\
& {\left[f_{n}-\frac{1}{k}<f\right]=\bigcup_{i=1}^{\infty} B(n, k, i)}
\end{aligned}
$$


where each of the sets $A(n, k, i), B(n, k, i)$ is closed. For all positive integers $n, k$, and $i$ define the sets

$$
H[A(n, k, i)]=\left\{(x, y): x \in A(n, k, i) \text { and } y \geq f_{n}(x)+\frac{1}{k}\right\},
$$

and

$$
H[B(n, k, i)]=\left\{(x, y): x \in B(n, k, i) \text { and } y \leq f_{n}(x)-\frac{1}{k}\right\} .
$$

It is easily checked that these sets are closed. Enumerate the set of all possible $H[A(n, k, i)]$ and $H[B(n, k, i)]$ as $\left\{H_{j}\right\}_{j=1}^{\infty}$. For each $n$ define $G_{n}=(X \times \mathbb{R}) \backslash$ $\bigcup_{j=1}^{n} H_{j}$. Then each $G_{n}$ is an open strip containing $f$. Given $x \in I$ and $\epsilon>0$ we can find $n_{1}, k_{1}, n_{2}$, and $k_{2}$ such that

$$
f(x)-\epsilon<f_{n_{2}}(x)-\frac{1}{k_{2}}<f(x)<f_{n_{1}}(x)+\frac{1}{k_{1}}<f(x)+\epsilon .
$$

Let $H_{s}=H\left[A\left(n_{1}, k_{1}, i_{1}\right)\right]$, where $x \in A\left(n_{1}, k_{1}, i_{1}\right)$, and $H_{t}=H\left[B\left(n_{2}, k_{2}, i_{2}\right)\right]$ where $x \in B\left(n_{2}, k_{2}, i_{2}\right)$. Then taking $j=\max \{s, t\}$ we have $\operatorname{rng}\left(G_{m}(x)\right) \subseteq$ $(f(x)-\epsilon, f(x)+\epsilon)$ whenever $m \geq j$. Hence, $\bigcap_{n=1}^{\infty} G_{n}=f$.

$(\Leftarrow)$ Let $f=\bigcap_{n=1}^{\infty} G_{n}$ where each $G_{n}$ is an open strip. If $X=I$ we can apply Lemma 2.1 , to pick $p_{n}$ to be a polygonal function in $G_{n}$ with $\operatorname{dom}\left(p_{n}\right)=I$. Using the fact that the sets $G_{m}(x)$ are intervals it is easy to show that $p_{n} \rightarrow f$ so that $f \in \mathcal{B}_{1}$. In the general case we can verify that for any open subset $V$ of $\mathbb{R}$ the set $\left\{x:\left(\operatorname{rng} G_{n}(x)\right) \cap V \neq 0\right\}$ is open and therefore by Michael's Selection Theorem [5] there exists a continuous function $f_{n}$ in $G_{n}$ with domain $X$.

Can we characterize important subclasses of $\mathcal{B}_{1}$ by means of strips so that the characterization is not simply a disguised version of the definition of the class? The answer is yes for $\mathcal{C}$, usc, and $\mathcal{D} \mathcal{B}_{1}$. First, as a reformulation of the fact that uniform limits of continuous functions are continuous, we have: $f$ is continuous iff $f$ is the intersection of a sequence of open strips whose vertical diameters tend to zero iff $f$ is the intersection of a sequence of open strips whose top and bottom boundaries are continuous functions. Here, the vertical diameter of a strip $G$ is $\sup \{$ length $G(x): x \in I\}$.

We say that an open strip $G$ is upper semicontinuous iff for each $\lambda \in \mathbb{R}$ the set $\{x: \operatorname{rng}(G(x)) \subseteq(-\infty, \lambda)\}$ is open, i.e., the strip $G$ behaves as though it were a usc function.

Theorem 2.3. A function $f$ is usc

if and only if $f$ is the intersection of a sequence of upper semicontinuous open strips, 
if and only if $f$ is the intersection of a sequence of open strips each of whose upper boundary is a continuous function.

Proof. Suppose $f$ is usc. Then there exists a sequence of continuous functions $\left\{f_{n}\right\}_{n=1}^{\infty}$ such that $f_{n} \downarrow f$. Proceed as in the proof of Theorem 2.2. Taking $H_{1}=H[A(1,1,1)]$ we see that each $G_{n}$ has a continuous function as its upper boundary and hence each $G_{n}$ is usc. The rest of the proof is easy.

Of course, there is a similar characterization for lower semicontinuous functions.

Theorem 2.4. A function $f$ is Darboux Baire 1 if and only if $f$ is the intersection of a bilaterally dense in itself sequence of open strips.

Proof. $(\Rightarrow)$ Let $\left\{W_{n}\right\}_{n=1}^{\infty}$ be a sequence of open strips, as constructed in the proof of Theorem 2.2, such that $f=\bigcap_{n=1}^{\infty} W_{n}$. Choose $\left\{\left(x_{k}, f\left(x_{k}\right)\right)\right\}_{k=1}^{\infty}$ to be a sequence dense in $f$. Define

$$
G_{n}=W_{n} \backslash \bigcup_{k=1}^{n}\left\{\left(x_{k}, y\right):\left|y-f\left(x_{k}\right)\right| \geq 1 / k\right\} .
$$

Then each $G_{n}$ is an open strip and $f=\bigcap_{n=1}^{\infty} G_{n}$. It remains to show that $\left\{G_{n}\right\}_{n=1}^{\infty}$ is bilaterally dense in itself. Let $B$ be any open square having some $G_{n}(x)$ in its left side. Choose $k$ such that $\overline{G_{k}(x)} \subseteq G_{n}(x)$. The set $B \cap$ $\left(I \times G_{k}(x)\right)$ contains infinitely many points $\left(x_{k_{i}}, f\left(x_{k_{i}}\right)\right), i=1,2,3, \ldots$ Since $G_{k_{i}}\left(x_{k_{i}}\right)$ has length less than $1 / k_{i}$, it follows that some $G_{k_{m}}\left(x_{m}\right) \subseteq B$. If $B$ has some $G_{m}(x)$ in its right side, the proof is analogous.

$(\Leftarrow)$ This part of the proof is simple and straightforward.

It is unknown whether in the proof of Theorem 2.4 one can replace the bilaterally dense in itself sequence of open strips by a sequence $\left\{G_{n}\right\}_{n=1}^{\infty}$ of open strips having the property that for each positive integer $n$, the set $\left\{G_{n}(x): x \in I\right\}$ is bilaterally dense in itself.

Although Theorems 2.3 and 2.4 are proven for a closed interval $I$ it is clear that their proofs can be adjusted to hold for any interval.

\section{The Main Result}

For a polygonal function $p$ we define mesh $(p)$ to be the length of the longest line segment of $p$ and $V(p)$ to be the set of vertices of $p$.

Theorem 3.1. A function $f$ is Darboux Baire 1 if and only if there is a sequence $\left\{p_{n}\right\}_{n=1}^{\infty}$ of polygonal functions having their vertices on $f$ such that mesh $\left(p_{n}\right) \rightarrow 0$ and $p_{n} \rightarrow f$. 
Proof. $(\Leftarrow)$ See the proof of Theorem 3.3, part $(\Leftarrow)$.

$(\Rightarrow)$ Let $\left\{G_{n}\right\}_{n=1}^{\infty}$ be a sequence of open strips such that $f=\bigcap_{n=1}^{\infty} G_{n}$, as in Theorem 2.2. Fix $n$, and for any closed interval $[c, d]$ define $\mathcal{P}[c, d]$ to be the set of all polygonal functions $p$ on $[c, d]$ such that $V(p) \subseteq f \mid[c, d]$ (i.e., $f$ restricted to $[c, d])$ and $p \subseteq G_{n}$ and mesh $(p)<1 / n$. Let $E=\{x \in I: \mathcal{P}[a, x] \neq \emptyset\}$.

First of all, $E \neq \emptyset$. To see this, pick an open disk $O$ centered at $(a, f(a))$ such that $O \subseteq G_{n}$. Since $f \in \mathcal{D}$ there is a point $(z, f(z))$ of $f$ in $O$ with $z>a$. Let $p$ be the line segment joining $(a, f(a))$ and $(z, f(z))$. Then $p \in \mathcal{P}[a, z]$, so $z \in E$. Now we need:

Lemma 3.2. Suppose that $T$ is a quadrilateral in $G_{n}$ with vertices $(z, f(w))$, $(w, f(w)),(w, \sigma)$ and $(z, f(z))$, where $z<w$ and $f(w)<\sigma \leq f(z)$, and assume that the non-vertical sides of $T$ have length less than $1 / n$. Then there exists $p$ in $\mathcal{P}[z, w]$ such that $p \subseteq T$ and mesh $(p)<1 / n$.

Proof. Let $L$ be the top side of $T$. Subdivide the right-hand side of $T$ into line segments of equal length by means of the points $(w, f(w))=\left(w, \mu_{k}\right)$, $\left(w, \mu_{k-1}\right), \ldots,\left(w, \mu_{1}\right)=(w, \sigma)$. Through each of these construct a line segment between the vertical sides of $T$ and parallel to $L$; select $k$ large enough that the parallelograms so formed have diameter less than $1 / n$. Let $L_{1}$ be the bottom edge of the topmost parallelogram. Since $f \in \mathcal{D B}_{1}, f$ is connected so $f$ must meet $L_{1}$; select $\left(x_{1}, f\left(x_{1}\right)\right) \in L_{1} \cap f$.

Next, consider the portion of the next-lower parallelogram lying to the right of the line $x=x_{1}$. Arguing as above, $f$ meets the bottom, $L_{2}$, of this parallelogram. Pick $\left(x_{2}, f\left(x_{2}\right)\right) \in L_{2} \cap f$. Proceeding, we get a sequence $\left\{\left(x_{i}, f\left(x_{i}\right)\right\}_{i=0}^{k}\right.$, where $\left(x_{0}, f\left(x_{0}\right)\right)=(z, f(z))$ and $\left(x_{k}, f\left(x_{k}\right)\right)=(w, f(w))$, such that $\left(x_{i}, f\left(x_{i}\right)\right), i \geq 1$ is on the bottom side of the $i$ 'th parallelogram and $x_{i}<x_{i+1}$ for all $i$. By construction the distance between successive points is less than $1 / n$. Let $q$ be the polygonal function joining these points, in order. Then $q \in \mathcal{P}[z, w]$ and mesh $(q)<1 / n$.

We return now to the proof of Theorem 3.1. In the sequel, the notation $S(u, v)$ will denote the line segment joining $(u, f(u))$ and $(v, f(v))$.

We shall show that $\lambda=\sup E$ belongs to $E$. Pick an open square $S \subseteq G_{n}$ of diagonal length less than $1 / n$ having $(\lambda, f(\lambda))$ as an interior point of its right side. Let $x=d$ be the equation of the left side of $S$. Pick a sequence $\left\{\left(x_{n}, f\left(x_{n}\right)\right)\right\}_{n=1}^{\infty}$ with $x_{n} \in E$ such that $x_{n} \rightarrow \lambda$. Without loss of generality we may assume that there exists an extended real number $\xi$ such that $f\left(x_{n}\right) \rightarrow \xi$. If $\xi$ belongs to the interior of the right side of $S$ then we apply Lemma 3.2 to get $\lambda \in E$. So we may assume that $\infty \geq \xi \geq \beta$, where the top side of $S$ is on the line $y=\beta$; further, suppose that no $\left(x_{n}, f\left(x_{n}\right)\right) \in \bar{S}$. Pick $x_{k} \in(d, \lambda)$ such that $f\left(x_{k}\right)>\beta$. Since $f \in \mathcal{D}$ there is a point $w$ in $\left(x_{k}, \lambda\right)$ such that 
$f(w)=\beta$. Choose $x_{m} \in(w, \lambda)$ with $f\left(x_{m}\right)>\beta$, and take $p$ in $\mathcal{P}\left[a, x_{m}\right]$. Let $L$ be the segment of $p$ which lies above $(w, f(w))$; denote by $(c, f(c))$ the left-hand endpoint of $L$. Then we have three cases.

Case $1 c \in[d, w)$.

Pick $p \in \mathcal{P}[a, c]$ and let $q=p \cup S(c, w) \cup S(w, \lambda)$. Then $q \in \mathcal{P}[a, \lambda]$ and mesh $V(q)<1 / n$.

Case $2 c<d$ and $\left(x_{k}, f\left(x_{k}\right)\right)$ lies on or above $L$.

Since $f \in \mathcal{D}, f$ hits $L$ over $\left(x_{k}, w\right)$ at some point $(u, f(u))$. Apply Lemma 3.2 to the trapezoid determined by the lines $x=u, x=w, y=f(w)$, and with top side the lower of $y=f(u)$ and $L$ restricted to $[u, w]$ to get $p \in \mathcal{P}[u, w]$. Choose $q \in \mathcal{P}[a, u]$. Then $h=q \cup p \cup S(w, \lambda)$ belongs to $\mathcal{P}[a, \lambda]$ and mesh $(h)<1 / n$.

Case $3 c<d$ and $\left(x_{k}, f\left(x_{k}\right)\right)$ lies below $L$.

Consider the figure $T$ formed by the lines $x=x_{k}, x=w, y=f(w)$, and with top side the lower of $y=f\left(x_{k}\right)$ and the line through $\left(x_{k}, f\left(x_{k}\right)\right)$ parallel to $L$. If this last side meets $y=\beta$ at a point between $x_{k}$ and $w$, then $T$ is a triangle and $S\left(x_{k}, w\right)$ has length less than $1 / n$. Take $p \in \mathcal{P}\left[a, x_{k}\right]$; then $h=p \cup S\left(x_{k}, w\right) \cup S(w, \lambda)$ is the desired polygonal function. If, on the other hand, the lower side does not meet $y=\beta$ between $x_{k}$ and $w$, then $T$ is a trapezoid and we can apply Lemma 3.2 to get $p \in \mathcal{P}\left[x_{k}, w\right]$. Pick $q \in \mathcal{P}\left[a, x_{k}\right]$. Then $h=p \cup q \cup S(w, \lambda)$ belongs to $\mathcal{P}[a, \lambda]$ and mesh $(h)<1 / n$.

Finally, to show that $\lambda=b$, assume that $\lambda<b$ and apply the argument used to show that $E \neq \emptyset$ to get a contradiction.

The proof of Theorem 3.1 with its heavy reliance on strips, suggests a more general problem: given any open neighborhood $G$ of an almost continuous function $f$, does there exist a polygonal function $p$ of arbitrarily small mesh whose vertices lie on $f$ ? (A function is almost continuous iff each open neighborhood of it contains a continuous function.) A Darboux Baire 1 function is almost continuous; moreover, an almost continuous function is connected. But neither of the reverse implications holds (see [1]). In particular an almost continuous function is not necessarily Baire 1 so an affirmative answer to the above question does not imply that there is a sequence of such polygonal functions approaching $f$ pointwise.

We can obtain a more general version of Therorem 3.1 by replacing the polygonal functions by suitable continuous functions. Roughly speaking, this 
version says that each $p_{n}$ gets sufficiently close to $f$ on an appropriately spaced finite set. More precisely:

Theorem 3.3. The function $f$ is Darboux Baire 1 if and only if there exists a sequence of continuous functions $\left\{f_{n}\right\}_{n=1}^{\infty}$ such that $f_{n} \rightarrow f$ and for each $n$ there exists a non-empty finite subset $F_{n}$ of $f_{n}$ such that $r_{n}=\max \left\{\operatorname{diam} f_{n} \mid J\right.$ : $J$ an interval, $\left.\left(f_{n} \mid J\right) \cap F_{n} \neq \emptyset\right\}$ and $s_{n}=\max \left\{\left|f_{n}(x)-f(x)\right|: x \in \operatorname{dom} F_{n}\right\}$ both tend to zero as $n \rightarrow \infty$.

Proof. $(\Rightarrow)$ This part of the proof is provided by Theorem 3.1 with $F_{n}=$ $V\left(p_{n}\right)$.

$(\Leftarrow)$ It suffices to show that $f$ is bilaterally dense in itself. Take $\epsilon>0$ and $z<b$ so that $\epsilon<b-z$, and consider the box $B=\{(x, y): 0<x-z<$ $\epsilon$ and $|y-f(z)|<\epsilon\}$. Pick $n$ so that $\left|f_{n}(z)-f(z)\right|<\epsilon / 4$ and both $r$ and $s$ are less than $\epsilon / 4$. Let

$$
c=\min \left\{z+\frac{\epsilon}{2}, \inf \left\{x: 0<x-z<\frac{\epsilon}{2} \text { and }\left|f_{n}(x)-f(z)\right|<\frac{\epsilon}{2}\right\}\right\} .
$$

Then $\operatorname{diam} f_{n} \mid(z, c) \geq \epsilon / 4$, so $F_{n} \cap\left(f_{n} \mid(z, c)\right) \neq \emptyset$. Take $\left(u, f_{n}(u)\right) \in F_{n}$ with $z<u<c$. Then $\left|f_{n}(u)-f(z)\right|<\epsilon / 2$ and $\left|f_{n}(u)-f(u)\right|<\epsilon / 4$. It follows that $|f(z)-f(u)|<\epsilon$ so that $(u, f(u))$ belongs to $B$. Hence, $f$ is bilaterally dense in itself, and the proof is complete.

We note that replacing the condition of Theorem 3.3 by saying that the Hausforff distance between $f_{n}$ and $f$ tends to zero, does not work. This can be seen by considering the non-Darboux function $f$, where $f(x)=\sin \frac{1}{x},-1 \leq$ $x<0, f(0)=0, f(x)=1,0<x \leq 1$.

We have been unable to find any applications of Theorems 3.1 and 3.3. One of the problems here is the lack of freedom in choosing the points in $\operatorname{dom} V\left(p_{n}\right)$ in Theorem 3.1, which renders the convergence in the sense of Theorem 3.1 deficient in many ways. For example, the convergence is not "additive": if $f_{n} \rightarrow f$ and $g_{n} \rightarrow g$ in the sense of Theorem 3.1, then it is not necessarily true that $f_{n}+g_{n} \rightarrow f+g$. This follows from the fact that the sum of two $\mathcal{D} \mathcal{B}_{1}$ functions can fail to be Darboux.

\section{The General Baire 1 Case}

One interesting question is whether or not other important subclasses of $\mathcal{B}_{1}$ have similar characterizations involving convergent sequences of polygonal functions. In case of $\mathcal{C}$ the answer is affirmative because continuity can be characterized by the condition of Theorem 3.1 with pointwise convergence replaced by uniform convergence. Here, the uniform convergence follows directly from $V\left(p_{n}\right) \subseteq f$ and $\operatorname{mesh}\left(p_{n}\right) \rightarrow 0$. 
The answer is also affirmative for the entire class $\mathcal{B}_{1}$, namely: each $\mathcal{B}_{1}$ function is a pointwise limit of a sequence of polygonal functions whose vertices lie on the function. (Theorem 4.1 below.) Note that this is just the characterizing condition of Theorem 3.1 without the mesh requirement.

In an earlier version of this paper we only were able to prove this result for some specific subclasses of $\mathcal{B}_{1}$ (e.g. usc and $\mathcal{B}_{1}^{*}$ ). One difficulty is that our technique of inserting polygonal functions into open strips breaks down in general. In fact one can easily construct a Baire 1 function $f$ with a 3 point range and an open strip containing $f$ which admits no polygonal function inside having vertices on $f$. So, if the insertion were to work it would at least require a delicate crafting of descending open strips.

Fortunately the referee came up with an easy proof based upon a deeper result involving first return continuity as follows:

Theorem 4.1. A function $f$ is Baire 1 if and only if there is a sequence of polygonal functions having their vertices on $f$ which converges to $f$ pointwise.

Proof. We need only show $(\Rightarrow)$. By Lemma 1 of Evans and O'Malley [3] if $f$ is Baire 1 then there exists a trajectory $\left\{x_{k}\right\}_{k=0}^{\infty}$ with respect to which $f$ is first return continuous at each point of $[0,1] \backslash\left\{x_{k}: k=0,1,2, \ldots\right\}$. (See [3] for the definitions of first return notions.) For each $n$ let $p_{n}$ be the polygonal function with vertices $(0, f(0)),(1, f(1)),\left(x_{0}, f\left(x_{0}\right)\right), \ldots,\left(x_{n}, f\left(x_{n}\right)\right)$. Let us show $p_{n}(z) \rightarrow f(z)$ for each $z$. If $z=x_{k}$ for some $k$ the result is obvious. For any other $z$ let $S_{n}$ be that segment in $p_{n}$ with $z \in \operatorname{dom} S_{n}=\left[a_{n}, b_{n}\right]$. It is clear that $a_{n}$ (resp. $b_{n}$ ) belongs to the left (resp. right) return path to $z$. Therefore the end points of $S_{n}$ approach $(z, f(z))$. Since $\left(z, p_{n}(z)\right)$ belongs to $S_{n}$ it follows that $p_{n}(z) \rightarrow f(z)$, completing the proof.

In conclusion, we present the referee's alternative proof of Theorem 3.1, which picks up where the proof of Theorem 4.1 left off:

Alternative Proof of Theorem 3.1. For the proof of the $(\Rightarrow)$ direction of Theorem 3.1 we continue, assuming further that $f$ is Darboux. All we need do is replace the polygonal sequence $\left\{p_{n}\right\}$ by another polygonal sequence $\left\{q_{n}\right\}$ which still converges to $f$ pointwise and for which mesh $q_{n} \rightarrow 0$. Since $\left\{x_{k}\right\}$ is a trajectory it is clear that the maximal horizontal distance between adjacent vertices of $p_{n}$ tends to zero as $n \rightarrow \infty$. We shall simply add finitely many vertices to each $p_{n}$, forming $q_{n}$ in such a way to guarantee that the maximal vertical distance between adjacent vertices of $q_{n}$ tends to zero as $n \rightarrow \infty$ as well, thus assuring that mesh $q_{n} \rightarrow 0$.

To this end, fix an $n$ and let $\left(a_{k}, f\left(a_{k}\right)\right)$ and $\left(b_{k}, f\left(b_{k}\right)\right)$ denote adjacent vertices of $p_{n}$. We may use the Darboux property of $f$ to select a finite set 
$D_{k}$ of points in $\left[a_{k}, b_{k}\right]$ so that if $y$ and $w$ are adjacent points in the set $D_{k} \cup\left\{a_{k}, b_{k}\right\}$, we have both that $f(y)$ and $f(w)$ are between $f\left(a_{k}\right)$ and $f\left(b_{k}\right)$ and that $|f(y)-f(w)|<1 / n$. We do this for each adjacent pair of vertices $\left(a_{k}, f\left(a_{k}\right)\right)$ and $\left(b_{k}, f\left(b_{k}\right)\right)$ in $p_{n}$ and form the polygonal function $q_{n}$ by using all the vertices of $p_{n}$ and all the newly selected vertices $(y, f(y))$ where $y \in D_{k}$ for some $k$. Clearly, mesh $q_{n} \rightarrow 0$.

Finally, if $z \in[0,1] \backslash\left\{x_{k}: k=0,1,2, \ldots\right\}$, using the notation of the first paragraph of this proof, we know that the end points of $S_{n}$ approach $(z, f(z))$, keeping in mind that $S_{n}$ is determined by $p_{n}$. But now, if we let $T_{n}$ be that segment in $q_{n}$ with $z \in \operatorname{dom} T_{n}=\left[c_{n}, d_{n}\right]$, we have that $\left[c_{n}, d_{n}\right] \subseteq\left[a_{n}, b_{n}\right]$ and that $f\left(c_{n}\right)$ and $f\left(d_{n}\right)$ each lie between $f\left(a_{n}\right)$ and $f\left(b_{n}\right)$. Consequently, the end points of $T_{n}$ approach $(z, f(z))$ as $n \rightarrow \infty$, completing the proof.

\section{References}

[1] J. B. Brown, Connectivity, semi-continuity and the Darboux property, Duke Math. J., 36 (1969), 559-562. MR 39 \#7568.

[2] J. G. Ceder and T. L. Pearson, A survey of Darboux, Baire 1 functions, Real Analysis Exchange, 9 (1983-84), 179-193. MR 86a:26003.

[3] M. J. Evans and R. J. O'Malley, Fine tuning the recoverability of Baire 1 functions, Real Analysis Exchange, 21 (1995-96), 165-174.

[4] F. B. Jones and E. S. Thomas, Jr., Connected $G_{\delta}$ graphs, Duke Math. J., 33 (1966), 341-346. MR 33 \#702.

[5] E. Michael, Continuous selections, Ann. Math., 63 (1956), 361-382. MR $17,990 \mathrm{e}$.

[6] E. S. Thomas, Some characterizations of functions of Baire class 1, Proc. Amer. Math. Soc., 17 (1966), 456-461. MR 32 \#6420. 